\title{
Seguridad y reproducibilidad de la cirugía colorrectal laparoscópica en dos centros académicos de tercer nivel en Sudamérica
}

\author{
Safeness and reproductivity of a laparoscopic colorectal program in two tertiary care \\ academic centers of South America
}

\author{
Martín Andrada*, Natalia P. Bollati, Franco J. Signorini, Pablo S. Maldonado, Federico Moser, \\ Lucio R. Obeide y Alejandro M.C. Rossini \\ Servicio de Cirugía General, Hospital Privado Universitario de Córdoba, Córdoba, Argentina
}

\begin{abstract}
Resumen
Objetivo: Evaluar la reproducibilidad y la seguridad de un programa de cirugía colorrectal laparoscópica en dos centros de Sudamérica. Método: Se realizó un estudio analítico-descriptivo. Se revisaron retrospectivamente los registros clínicos de pacientes sometidos a cirugía videolaparoscópica colorrectal desde el año 2012 hasta el año 2018, en dos centros académicos de tercer nivel argentinos. Se analizaron datos demográficos, indicaciones y tiempos quirúrgicos, tasa de conversión, evolución posoperatoria, morbimortalidad y resecabilidad oncológica, y se comparó con el abordaje convencional. Resultados: Se realizaron 505 cirugías. La edad media de los pacientes fue de 63.4 años y el 50.9\% eran hombres. El tiempo operatorio medio fue de 175 minutos. La principal indicación fue cáncer de colon. La incidencia de conversión fue del 9.5\%. El promedio de ganglios por pieza quirúrgica en patología neoplásica fue de 15.9. La morbilidad fue del 35.4\%, en su mayoría complicaciones menores. La tasa de fístulas fue del 11.7\%. La mortalidad a 30 días fue del 2.5\%. Conclusión: La cirugía colorrectal laparoscópica podría representar una opción segura y reproducible en un centro de tercer nivel de un país en desarrollo.
\end{abstract}

Palabras clave: Cáncer colorrectal. Cirugía laparoscópica. Colon. Recto.

\begin{abstract}
Objective: To evaluate the feasibility and safeness of a colorectal laparoscopic program in two centers form South America. Method: We retrospectively review the records of patients who underwent laparoscopic colorectal surgery from 2012 to 2018 in two tertiary care academic centers. Surgical indication, operative time, conversion rate, lymph nodes harvested, surgical margins and complications were analyzed. This results were then compared to the open approach. Results: We collected data from 505 patients, mean age 63.4, 50.9\% male. The most frequent indication was colon cancer, mean operative time was 175 minutes. Conversion rate was $9.5 \%$, mean nodes harvested was 15.9 with free resection margins in every case. Morbidity was $35.4 \%$ at 30 days, most of them were minor complications. The leak rate was $11.7 \%$. The 30-day mortality was $2.5 \%$. Conclusion: The laparoscopic approach for colorectal surgery might represent a safe and feasible option in an tertiary care hospital from a developing country.
\end{abstract}

Key words: Colorectal cancer. Laparoscopic surgery. Colon. Rectum.

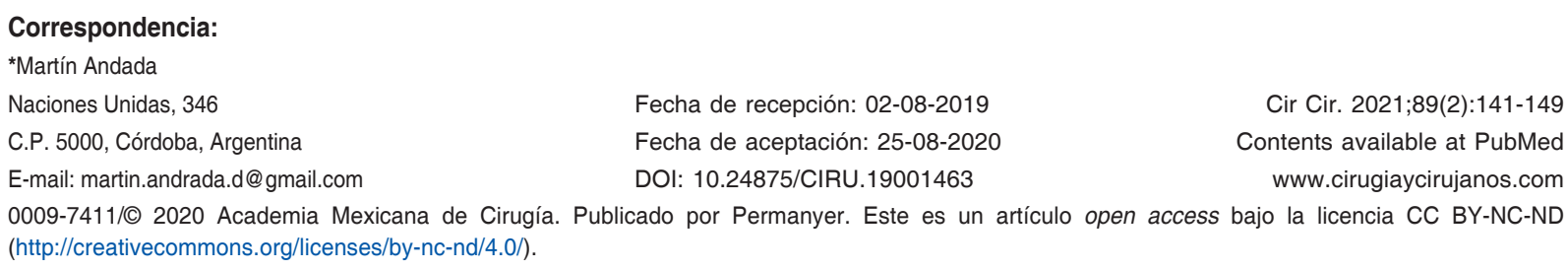




\section{Introducción}

Desde que la videolaparoscopia se utilizó por primera vez en la colecistectomía en $1985^{1}$, se ha desarrollado rápidamente y se ha utilizado en la mayoría de los campos de la cirugía tradicional.

La primera colectomía videolaparoscópica fue realizada en 1991 por Jacobs, et al. ${ }^{2}$ en Miami, Florida. Desde entonces, numerosos estudios controlados aleatorizados han demostrado la asociación de la cirugía laparoscópica colorrectal con menor estancia hospitalaria, menos dolor posoperatorio y recuperación temprana del tránsito intestinal en comparación con la vía abierta ${ }^{3-10}$.

Sin embargo, la aceptación de la técnica como método de tratamiento para la patología colorrectal no ha sido la misma en comparación con otros procedimientos abdominales laparoscópicos (colecistectomía, cirugía de Nissen, apendicetomía, adrenalectomía, etc.). Este fenómeno se sustenta en una curva de aprendizaje mayor, unos tiempos operatorios más largos, la necesidad de un abordaje periquirúrgico multidisciplinario y unas complicaciones más graves ${ }^{11,12}$. Por otra parte, inicialmente hubo suspicacias respecto a la seguridad oncológica del procedimiento ${ }^{13-15}$.

Actualmente, numerosos estudios prospectivos aleatorizados y multicéntricos han demostrado que no hay diferencias en cuanto a resultados a corto y largo plazo, radicalidad oncológica, supervivencia global, supervivencia libre de enfermedad y tasas de recurrencia cuando se compara el abordaje laparoscópico con el convencional ${ }^{16-28}$. Sin embargo, los resultados encontrados en la literatura provienen de centros de primer nivel mundial, con amplio acceso a recursos materiales y humanos, que generan una contingencia no siempre replicable en los países en desarrollo.

El objetivo de este estudio es reportar la factibilidad, la seguridad y los resultados oncológicos, así como la morbilidad y la mortalidad, de la cirugía colorrectal laparoscópica en dos centros académicos de tercer nivel de un país en desarrollo.

\section{Método}

Se realizó un estudio retrospectivo, analítico-descriptivo, en el que se revisaron los registros clínicos de pacientes sometidos a cirugía colorrectal laparoscópica en el Hospital Privado Universitario de Córdoba y en el Hospital Raúl A. Ferreyra, en el periodo comprendido entre enero de 2012 y octubre de 2018.
Se incluyeron todos los pacientes operados por vía laparoscópica de manera consecutiva en el periodo mencionado, considerando cirugías programadas 0 de guardia. Los criterios de exclusión para la cirugía laparoscópica fueron: 1) inestabilidad hemodinámica; 2) perforación intestinal con peritonitis generalizada; 3) abdomen hostil por gran compromiso adherencial; 4) contraindicación anestésica; 5) tumor adherido o fistulizado a órganos o vísceras vecinas (T4); y 6) preferencia del cirujano o del paciente.

Las variables analizadas fueron: 1) demográficas (sexo, edad, índice de masa corporal [IMC], comorbilidad categorizada de acuerdo con la American Society of Anesthesiologists $[A S A]^{29}$ ); 2) preoperatorias (indicación quirúrgica y distribución entre patologías benignas y malignas); y 3) intraoperatorias (tipo de procedimiento, tiempo quirúrgico, complicación intraoperatoria y tasa de conversión). Además, se estudió la evolución de la indicación del abordaje laparoscópico en relación con el convencional a lo largo de los años. Por otro lado, se valoraron variables posoperatorias, desde la cirugía hasta los 30 días posteriores, como la tolerancia oral, la morbilidad, la estancia hospitalaria, la mortalidad y las tasas de reintervención y de readmisión. Las complicaciones posoperatorias se graduaron según la clasificación de Clavien Dindo ${ }^{30}$; se consideraron complicaciones menores los grados I y II y complicaciones mayores las correspondientes a los grados III y IV. Por último, se analizó también la tasa de fistula, en la que se incluyó la aparición de fuga anastomótica, sepsis abdominal y abscesos intraabdominales. En los procedimientos por enfermedad maligna se evaluaron los márgenes quirúrgicos y la cantidad de ganglios resecados.

Se definió conversión como la situación en la cual se debió realizar una incisión mayor que la requerida para extraer la pieza quirúrgica o para realizar maniobras en forma convencional.

Por último, se compararon los resultados obtenidos en esta muestra con nuestra casuística por vía convencional, sin excluir ningún paciente. Ambos grupos se compararon estadísticamente mediante la prueba de ji al cuadrado para variables cualitativas y mediante ANOVA para las cuantitativas. Se utilizó el programa SPSS (IBM SPSS Statistics 25.0, Chicago, IL) para el procesamiento de los datos.

\section{Resultados}

Se realizaron 505 cirugías colorrectales por videolaparoscopia en el tiempo comprendido entre enero 


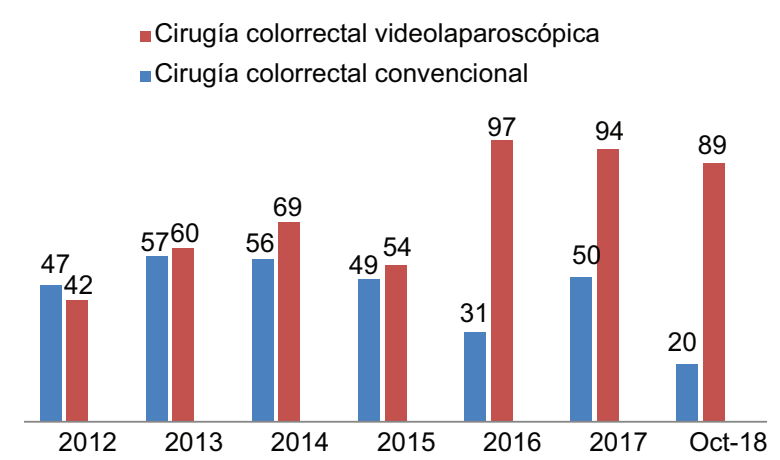

Figura 1. Cirugías colorrectales por año.

de 2012 y octubre de 2018. La edad media de los pacientes fue de 63.4 años (rango: 15-89), el 50.9\% eran hombres y el IMC promedio fue de 27.4. La mayoría de los pacientes fueron ASA II (66.1\%).

En el año 2012 se realizaron por videolaparoscopia el 47\% de las cirugías colorrectales, y en el año 2018 fueron el 81\% (Fig. 1). En 113 casos (22.3\%) la cirugía fue indicada por procesos benignos y en 392 casos (77.6\%) por neoplasias (Tabla 1). El $73.8 \%$ de los procedimientos fueron en el colon y el $26.1 \%$ incluyeron resección del recto (Tabla 2). El tiempo operatorio medio fue de 175.4 minutos (rango: 47-560).

La incidencia de complicaciones intraoperatorias fue del $1.9 \%$ (en 10 procedimientos); en seis casos se precisó conversión para su resolución. La incidencia global de conversión fue del 9.5\% (48 casos), en su mayoría por adherencias excesivas o por mala exposición debido a obesidad intraabdominal (Tabla 3). La necesidad de conversión se asoció de manera estadísticamente significativa con mayor morbilidad y más mortalidad ( $p$ $=0.017$ y $p=0.002$, respectivamente) (Tabla 4).

La morbilidad global de la serie fue del 35.4\% (228 complicaciones en 179 pacientes), en su mayoría complicaciones menores: 132 complicaciones Clavien Dindo I y || en 112 pacientes. Entre ellas, las más frecuentes fueron 38 infecciones de herida quirúrgica $(7.5 \%), 31$ fístulas que se resolvieron con tratamiento médico (6.3\%),12 íleos (2.3\%), 11 infecciones del tracto urinario $(2.1 \%)$ y 7 casos de alto débito por la ileostomía (1.3\%).

La incidencia de complicaciones mayores fue del 13.8\%: 70 pacientes con complicaciones Clavien Dindo III y IV (Fig. 2). La tasa global de fístulas fue del $11.7 \%$ (59 casos); 34 (58\%) correspondieron a complicaciones menores, y de estas, 27 fueron fístulas dirigidas con resolución espontánea y 7 colecciones que se resolvieron con tratamiento antibiótico. Las
Tabla 1. Indicaciones quirúrgicas

\begin{tabular}{lcc}
\hline Patologías & Pacientes (n) & $\%$ \\
\hline Cáncer de colon-recto & 392 & 77.6 \\
Pólipos de colon-recto & 60 & 11.8 \\
Enfermedad diverticular & 40 & 7.92 \\
Otros & 13 & 2.5 \\
\hline
\end{tabular}

Tabla 2. Procedimientos quirúrgicos realizados

\begin{tabular}{lcc}
\hline Procedimientos & $\mathbf{n}$ & $\%$ \\
\hline Hemicolectomía izquierda & 154 & 30.5 \\
Sigmoidectomía & 51 & 10 \\
Hemicolectomía derecha & 149 & 29.5 \\
Hemicolectomía derecha ampliada & 19 & 3.7 \\
Colectomía total & 8 & 1.6 \\
Resección anterior baja & 107 & 21.2 \\
Resección abdominal-perianal & 17 & 3.3 \\
\hline
\end{tabular}

Tabla 3. Causas e incidencia de conversión

\begin{tabular}{lcc}
\hline Causa & $\mathbf{n}$ & $\%$ \\
\hline Adherencias del tumor a tejidos vecinos & 19 & 39.5 \\
$\begin{array}{l}\text { Mala exposición (obesidad intraabdominal, colon } \\
\text { redundante) }\end{array}$ & 21 & 43.7 \\
Adherencias por cirugías previas & 3 & 6.2 \\
Lesión arterial, sangrado incoercible & 3 & 6.2 \\
Isquemia colónica & 1 & 2 \\
Variación anatómica & 1 & 2 \\
\hline
\end{tabular}

Tabla 4. Morbimortalidad asociada a la conversión

\begin{tabular}{lcccc}
\hline & $\mathbf{n}$ & $\begin{array}{c}\text { Morbilidad } \\
\text { global }\end{array}$ & $\begin{array}{c}\text { Morbilidad } \\
\text { mayor }\end{array}$ & Mortalidad \\
\hline No convertido & 457 & $36.7 \%(166)$ & $12.6 \%(58)$ & $1.9 \%(9)$ \\
Convertido & 48 & $60.4 \%(29)$ & $20.8 \%(10)$ & $8.3 \%(4)$ \\
$p$ & & 0.017 & & 0.002 \\
\hline
\end{tabular}

complicaciones mayores secundarias a fístulas anastomóticas representaron un $4.9 \%$ del total (25 casos): 6 colecciones con necesidad de drenaje percutáneo, 


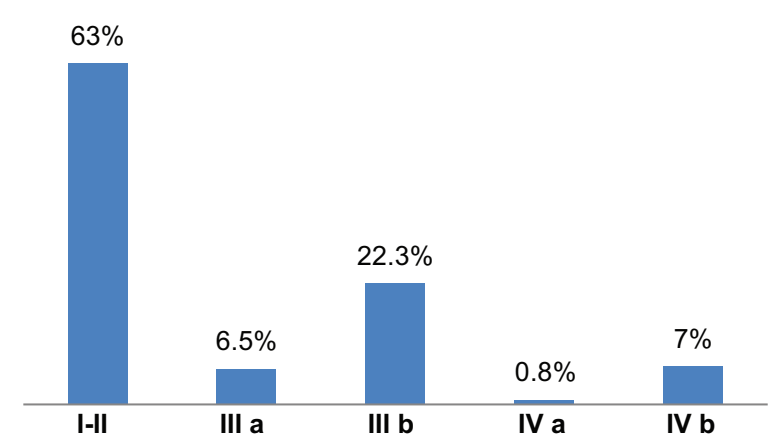

Figura 2. Porcentaje de complicaciones según la clasificación de Clavien-Dindo.

14 fístulas con necesidad de reintervención, de las cuales 2 precisaron posoperatorio en unidad cerrada con necesidad de vasopresores y en asistencia respiratoria mecánica (4 lavados y colocación de drenaje, 6 conversión a Hartmann, 3 rafias, 1 caso en que se rehízo la anastomosis), y 5 casos que precisaron recirugía y culminaron en el óbito del paciente.

Se reoperaron 49 pacientes $(9.7 \%$ ) y la tasa de reinternación a los 30 días fue del 7.7\% (39 pacientes) (Tablas 5 y 6 ).

La mortalidad a 30 días de esta serie fue del 2.5\% (13 pacientes): 5 casos de dehiscencia de anastomosis, 1 caso de sangrado en un paciente coagulopático con hepatopatía crónica, 1 caso de isquemia de intestino delgado secundario a lesión en la raíz de la vena mesentérica superior, 1 paciente que reingreso al hospital en shock séptico y no pudo encontrarse un foco infeccioso de origen, 2 casos de neumopatía, 1 paciente que presentó un paro cardiorrespiratorio en el posoperatorio mediato estando en condiciones de alta hospitalaria, y 2 pacientes que presentaron accidente cerebrovascular.

Con respecto a la recuperación de la función intestinal, el primer flato ocurrió después de 1.5 días, el primer movimiento intestinal se produjo a los 1.7 días y la tolerancia de la ingesta oral se logró a los 2.5 días.

La estadía hospitalaria global fue en promedio de 8.2 días, con un rango de 2 a 69 días; para pacientes con complicaciones mayores fue de 16.7 días en promedio y para pacientes no complicados fue de 5.7 días en promedio.

En cuanto a la linfadenectomía quirúrgica, en los casos de cirugía por enfermedad maligna el promedio de ganglios extraídos por pieza quirúrgica fue de 15.9 \pm 9.6 . En todos los casos se lograron márgenes quirúrgicos distales libres de enfermedad. No se registró
Tabla 5. Causas de reoperaciones

\begin{tabular}{lcc}
\hline Causas & $\mathbf{n}$ & $\%$ \\
\hline Fístula o dehiscencia de anastomosis & 19 & 38.7 \\
Sangrado & 10 & 20.4 \\
Hernia interna & 3 & 6.1 \\
Evisceración & 6 & 12.2 \\
Plástica de ileostomía & 2 & 4 \\
Perforación de ID por lesión inadvertida & 2 & 4 \\
Necrosis y dehiscencia de ostoma & 3 & 6.1 \\
Vólvulo a nivel de ileostomía & 1 & 2 \\
Isquemia intestinal & 2 & 4 \\
Toilette de IHQ perianal & 1 & 2 \\
Total & 49 & $9.7 \%$ \\
\hline
\end{tabular}

ID: intestino delgado; ISQ: infección de sitio quirúrgico.

Tabla 6. Causas de reinternación

\begin{tabular}{lcc}
\hline Causas & $\mathbf{n}$ & $\%$ \\
\hline Fístula & 12 & 30.7 \\
Deshidratación por alto débito por ostoma & 5 & 12.8 \\
íleo & 5 & 12.8 \\
Hemorragia digestiva baja autolimitada & 4 & 10.2 \\
Evisceración & 3 & 7.7 \\
Infección del tracto urinario & 3 & 7.7 \\
Fiebre sin foco & 3 & 7.7 \\
Fecaloma & 1 & 2.5 \\
Fístula uretral & 1 & 2.5 \\
Vólvulo delgado & 1 & 2.5 \\
Hernia interna & 1 & 2.5 \\
Total & 39 & $7.7 \%$ \\
\hline
\end{tabular}

recurrencia de la enfermedad en los sitios de los trocares laparoscópicos.

En el grupo de cirugía convencional se realizaron 324 intervenciones colorrectales en el tiempo establecido. La edad media de la población fue de 65.7 años (rango: $18-91$ ), el $58.6 \%$ eran hombres y el IMC promedio fue de 28.3. La mayoría de los pacientes (48.9\%) fueron ASA 3. No se establecieron diferencias significativas con respecto al grupo de cirugía laparoscópica. 
En 92 casos (28.4\%) la cirugía fue indicada por procesos benignos y en 232 casos $(71.6 \%)$ por neoplasias ( $p$ no significativa). El tiempo operatorio medio fue de 188.1 minutos (rango: $30-600)(p=0.03$ ).

La morbilidad global de la serie fue del $60.8 \%$ (presentaron complicaciones 197 pacientes). Al comparar este resultado con el obtenido en el grupo de cirugía laparoscópica, la diferencia fue significativa $(p=0.001)$. Al analizar las complicaciones menores, 101 pacientes presentaron complicaciones Clavien Dindo I y II. Entre ellas, las más frecuentes fueron 63 infecciones de herida quirúrgica (19.5\%), 14 fístulas que se resolvieron con tratamiento médico (4.3\%), 11 íleos (3.4\%) y 20 infecciones del tracto urinario (6.1\%).

La incidencia de complicaciones mayores fue del 31.5\%; 102 pacientes presentaron complicaciones Clavien Dindo III y IV. Al comparar con el grupo de cirugía laparoscópica, la diferencia fue significativa $(p=0.002)$. La tasa de fístulas que generaron complicaciones mayores fue del 4.3\% (14 casos), menor que en el grupo de cirugía laparoscópica, sin diferencia estadísticamente significativa $(p=0.67)$.

Fueron reoperados el $14.9 \%$ de los pacientes, frente al $9.7 \%$ en el grupo de cirugía laparoscópica ( $p=0.02$ ), y la tasa de reinternación a los 30 días fue del $8.7 \%$ frente al $7.8 \%$, sin significancia estadística $(p=0.63)$.

La mortalidad a 30 días de esta serie fue del $5.9 \%$ (19 pacientes), con una diferencia estadísticamente significativa respecto al grupo de cirugía laparoscópica $(p=0.01)$.

La estadía hospitalaria global fue en promedio de 12.8 días \pm 12.1 , con una diferencia estadísticamente significativa respecto al grupo de cirugía laparoscópica $(p=0.01)$.

\section{Discusión}

La resección colorrectal laparoscópica ha experimentado un importante desarrollo desde el primer caso reportado en 1991, evolucionando hasta convertirse en el abordaje de elección por muchos cirujanos colorrectales. Sin embargo, la aplicación generalizada de esta técnica fue obstaculizada inicialmente por la pronunciada curva de aprendizaje y las preocupaciones sobre la seguridad oncológica ${ }^{11-15}$. En los últimos años, los resultados de varios estudios aleatorizados han confirmado la seguridad oncológica de la colectomía laparoscópica para el cáncer, y además han demostrado que los resultados a largo plazo son equivalentes a los de la cirugía abierta ${ }^{18-28}$. En todo el mundo ha habido un incremento en las cirugías colorrectales laparoscópicas realizadas, y lo mismo ha sucedido en nuestra institución en los últimos años ${ }^{31-33}$.

Nuestro grupo comenzó a realizar en forma electiva cirugía laparoscópica colorrectal a partir del año 2008, de manera esporádica, aumentando el número de casos y la complejidad paulatinamente. Desde el año 2012, con la implementación de la historia clínica electrónica, se mantiene un registro preciso de todas las cirugías que realiza el servicio, lo que facilita la recolección de datos.

La adaptación a la cirugía laparoscópica colorrectal demanda una compleja curva de aprendizaje multidisciplinario, y de inicio no es aplicable a todo tipo de pacientes; no obstante, es factible ampliar el espectro de indicaciones a medida que se avanza en el número de pacientes operados. En nuestro caso, el número de pacientes operados anualmente aumentó de manera progresiva hasta alcanzar en la actualidad un $81 \%$ de las intervenciones colorrectales. Este incremento se puede atribuir a una mayor aceptación del procedimiento por parte de los profesionales de la salud, un mayor número de cirujanos entrenados y el conocimiento público de las ventajas de la cirugía mínimamente invasiva. Además, ambos son procedimientos eficaces y una técnica no invalida a la otra; la elección del procedimiento más adecuado dependerá del profesional, de su experiencia, de las características del paciente y de su patología.

En esta serie de un periodo de 7 años, desde 2012 hasta 2018, pudimos observar que las resecciones colorrectales laparoscópicas realizadas en dos centros académicos de tercer nivel en Sudamérica pueden tener resultados aceptables en el corto plazo. Aunque el número de pacientes incluidos en nuestra serie es similar al de los grandes estudios prospectivos aleatorizados ${ }^{5,7,9,18,34}$, el nuestro es un estudio que presenta la limitación de su naturaleza retrospectiva. Sin embargo, por ser una serie consecutiva de pacientes y haber incluido todas las cirugías laparoscópicas de colon y recto, programadas o de guardia, realizadas por toda patología colorrectal, el sesgo de selección se reduce.

El tiempo medio quirúrgico de 175 minutos se ubicó dentro del rango de otros estudios de ámbito internacional, que reportan aproximadamente 142 a $268 \mathrm{mi}-$ nutos. Por otro lado, se obtuvo un resultado similiar al analizar el tiempo de restitución del tránsito intestinal y la estadia hospitalaria ${ }^{5-9,18,23,34}$. Estos tiempos no mostraron marcadas diferencias con los obtenidos en el grupo de cirugía convencional. El tiempo 
operatorio mostró alguna ventaja estadística con respecto al grupo de cirugía laparoscópica que podría ser explicada por los tiempos de laparotomía y cierre, el mayor número de cirugías de guardia u oncológicamente avanzadas en el grupo convencional y también por la mayor participación de cirujanos no especializados en la patología colorrectal y residentes, lo que se corresponde con el perfil académico de las instituciones participantes.

La morbilidad posoperatoria en cirugía colorrectal oscila, según las numerosas publicaciones, entre el 11 y el $47.9 \%^{3-10,18,23,34 ;}$; estas grandes variaciones se deben fundamentalmente a criterios heterogéneos de inclusión, seguimiento y definiciones de las distintas complicaciones. En nuestra serie, la morbilidad global posoperatoria a 30 días fue del $35.4 \%$. Si bien este porcentaje puede ser considerado elevado, cabe aclarar que el $63 \%$ fueron complicaciones menores que respondieron al tratamiento médico y no requirieron niguna intervención quirúrgica endoscópica o radiológica para su resolución. El grupo de cirugía convencional presentó un número mayor de complicaciones, pero esto se encuentra asociado a la mayor complejidad de los casos en este grupo.

La fuga anastomótica después de la cirugía colorrectal es una frecuente y temida complicación que resulta en una morbilidad y una mortalidad aumentadas $^{35,36}$. No podemos dejar de mencionar que esta fue la complicación con que nos encontramos de forma más frecuente, conformando también la principal causa de reintervención y de óbito. Si bien la incidencia de fístula del $11.7 \%$ reportada en esta serie puede considerarse relativamente alta, la mayoría de los reportes solo incluyen las dehiscencias de anastomosis y evalúan la tasa de abscesos intraabdominales o de sepsis abdominal por separado, al mismo tiempo que no todos incluyen cirugías rectales ${ }^{6,7,18,23}$. Denost, et al. $^{34}$ incluyen como fístula anastomótica criterios similares a los expuestos en este trabajo, resultando en una incidencia de fuga del $17 \%$; sin embargo, en esta serie solo se incluyen resecciones rectales y esto explicaría el número más elevado. Por otra parte, siendo un trabajo retrospectivo, nuestro estudio incluyó patología tanto colónica como rectal, cirugías programadas y de guardia, lo que genera una muestra menos homogénea. Sin embargo, la objetivación de los resultados de esta experiencia aporta un punto de referencia que no se encuentra descrito para la región en este tipo de cirugías. Por otro lado, la inclusión consecutiva de todos los pacientes intervenidos también aporta una referencia real de los resultados

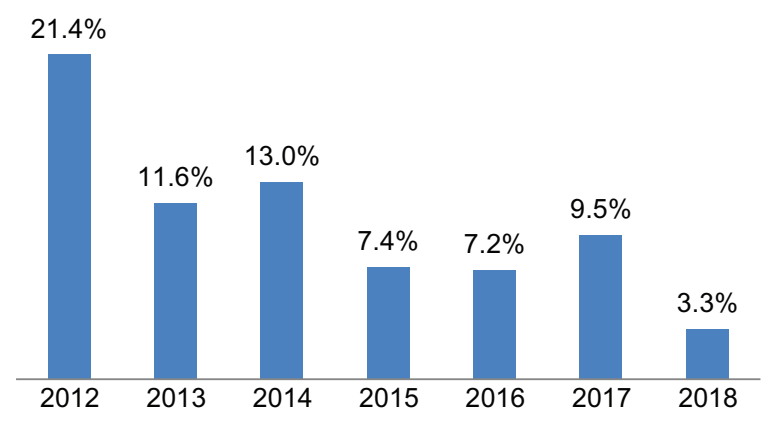

Figura 3. Tasa de conversión por año.

obtenidos a lo largo de nuestra experiencia, que podría servir a otros centros inmersos en realidades sanitarias similares al implementar este tipo de programas.

Se ha sugerido que una baja tasa de conversión contribuye a una morbilidad operatoria reducida; si bien la conversión a cirugía abierta no es una complicación en sí misma, se debe tener en cuenta porque los beneficios de la cirugía laparoscópica disminuyen a medida que aumenta la tasa de conversión ${ }^{37}$. Un análisis de subgrupos realizado por los autores del estudio COST mostró que los pacientes que requirieron conversión experimentaron más complicaciones que aquellos cuya cirugía se había completado por laparoscopia (7.8 vs. 2.9\%). La supervivencia general a 5 años también fue menor en estos pacientes (69 vs. $80 \%)^{38}$. En el estudio CLASICC, la cirugía convertida se asoció con una estancia hospitalaria de hasta 2 semanas más que la laparoscópica, y con una tasa de complicaciones más alta (93 vs. $50 \%$ después de cirugía abierta y $59 \%$ después de la laparoscópica) ${ }^{5}$. En los estudios incluidos en esta revisión, las tasas de conversión variaron del 5 al 33\%, mientras que en nuestra serie fue del $9.5 \%^{3-10,18,23,28}$. En nuestra experiencia, pudimos observar la elevada morbimortalidad asociada a la conversión, coincidiendo con la literatura ${ }^{37,38}$ (Tabla 4). En el estudio CLASICC, la tasa de conversión a cirugía abierta para el cáncer colorrectal disminuyó a medida que se adquirió más experiencia: del $38 \%$ en el primer año al $16 \%$ en el sexto año ${ }^{5}$. Este mismo fenómeno se puede ver en nuestra serie (Fig. 3). No dudamos de que la conversión temprana, en los casos en que no se progresa con el procedimiento o nos encontramos ante complicaciones intraoperatorias difíciles de resolver, debe ser la conducta a seguir para reducir el elevado riesgo de complicaciones que conlleva la 
Tabla 7. Comparación de resultados con los principales estudios internacionales

\begin{tabular}{|c|c|c|c|c|c|c|c|c|c|}
\hline & BRAGA & BARCELONA & Cost & COLOR & CLASICC & COLOR II & GRECCAR 5 & LEUNG & HP-HF \\
\hline $\begin{array}{l}\text { Número de cirugías } \\
\text { laparoscópicas }\end{array}$ & 136 & 105 & 435 & 536 & 526 & 699 & 469 & 203 & 505 \\
\hline Exclusión & $\begin{array}{c}\text { Guardia, transverso, } \\
\text { patología benigna }\end{array}$ & $\begin{array}{c}\text { Guardia, recto, } \\
\text { transverso, } \\
\text { patología benigna }\end{array}$ & $\begin{array}{c}\text { Guardia, recto, } \\
\text { transverso, } \\
\text { patología benigna }\end{array}$ & $\begin{array}{c}\text { Guardia, recto, } \\
\text { transverso, } \\
\text { patología } \\
\text { benigna }\end{array}$ & $\begin{array}{c}\text { Guardia, } \\
\text { transverso, } \\
\text { patología benigna }\end{array}$ & $\begin{array}{c}\text { Más de } 15 \mathrm{~cm} \\
\text { de margen anal, } \\
\text { guardia, patología } \\
\text { benigna }\end{array}$ & $\begin{array}{c}\text { Más de } 15 \mathrm{~cm} \text { margen } \\
\text { anal, Miles, guardia, } \\
\text { patología benigna }\end{array}$ & $\begin{array}{c}\text { Guardia, recto } \\
\text { bajo, patología } \\
\text { benigna }\end{array}$ & $\begin{array}{c}\text { Abdomen } \\
\text { hostil, tumor } \\
\text { T4, perforación } \\
\text { intestinal }\end{array}$ \\
\hline Tiempo quirúrgico (min) & 222 & 142 & 150 & 145 & 180 & 240 & 268 & 190 & 174 \\
\hline Tasa de conversión (\%) & 5,1 & 11 & 21 & 17 & 29 & 17 & - & 23.3 & 9.5 \\
\hline Estadía hospitalaria (días) & 10.4 & 5.2 & 5 & 8.2 & 9 & 8 & 12 & 8.2 & 8.4 \\
\hline Ganglios resecados & 14.8 & 11 & 12 & 10 & 13 & 13 & - & 11.1 & 15.9 \\
\hline Márgenes positivos (\%) & - & - & 5 & 2 & 6 & 10 & - & - & 0 \\
\hline Morbilidad (\%) & 20.6 & 11 & 21 & 21 & 33 & 40 & 47.9 & 19.7 & 35.4 \\
\hline Mortalidad (\%) & 0.7 & 0.9 & 0.5 & 1 & 4 & 1 & 1.1 & 2.4 & 2.4 \\
\hline Fístula (\%) & 5.9 & - & - & 3 & 10 R 3 C & 13 & 17.1 & 0.98 & 11.7 \\
\hline Reintervención (\%) & 5.9 & - & 2 & 7 & - & 16 & 18 & 2.9 & 9.7 \\
\hline
\end{tabular}


conversión. La laboriosidad del procedimiento y el tiempo prolongado provocan la fatiga del equipo quirúrgico y lo expone más fácilmente a lesiones, pero sobre todo a la toma de decisiones equivocadas sesgadas por el agotamiento intraoperatorio. Finalmente, la mortalidad durante los primeros 30 días posoperatorios varía entre el 0.5 y el $4 \%$ en la literatura revi-

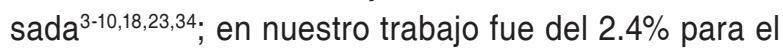
grupo de cirugía laparoscópica. Otra vez, el grupo de cirugía convencional mostró un resultado empobrecido por la complejidad de los casos incluidos.

El mayor número de metástasis en la pared abdominal, y en particular en los sitios de los trocares laparoscópicos, descrito en algunas de las primeras series publicadas en la década de 1990, causó gran preocupación con respecto a la indicación de técnicas mínimamente invasivas en el tratamiento de tumores gastrointestinales ${ }^{13-15}$. Sin embargo, los ensayos controlados aleatorizados posteriores han mostrado consistentemente una incidencia muy baja de recurrencia en el sitio del puerto en el grupo de cirugía laparoscópica, disipando efectivamente este temor inicial. En nuestra serie no se registraron metástasis en los sitios de los trocares laparoscópicos, colaborando con los resultados ya reportados sobre este punto. En la actualidad, la evidencia también ha demostrado que no existe una diferencia significativa entre el número de ganglios linfáticos examinados y los márgenes de resección entre los métodos laparoscópicos y los convencionales de cirugía abierta ${ }^{18-28}$. La mayoría de los casos de nuestra serie (76\%) fueron operados por enfermedad maligna. En estos pacientes, logramos alcanzar un promedio global de resección de 15.9 ganglios linfáticos y no obtuvimos márgenes comprometidos, lo que sugiere que es factible realizar un abordaje oncológicamente seguro, consistente con el mínimo recomendado de 12 ganglios linfáticos para una estadificación precisa.

Los resultados de una serie quirúrgica pueden presentar variaciones secundarias a la diversidad de criterios para algunos conceptos propios del paciente, el procedimiento, las complicaciones y hasta del mismo cirujano. Por otra parte, la extrapolación directa de los resultados de las experiencias del primer mundo hacia centros de países en desarrollo puede establecer una vara difícil de alcanzar debido a las conocidas diferencias de acceso a los recursos. Esta vara, incluso, puede representar un riesgo en sí misma, ya que puede predisponer a un intento de replicar procesos sin los medios adecuados y, por tanto, arriesgando al paciente. Debido a esto, resulta importante para cada servicio quirúrgico conocer los resultados de su propia experiencia, con los recursos y la población en que se encuentre inmerso (Tabla 7).

\section{Conclusión}

A la luz de estos resultados, la cirugía colorrectal laparoscópica podría representar una opción segura y reproducible en un centro de tercer nivel de un país en desarrollo siempre que se conozcan sus propios resultados al respecto. Es posible que los estudios prospectivos multicéntricos realizados en países subdesarrollados puedan esclarecer esta cuestión.

\section{Financiamiento}

Los autores no recibieron patrocinio para llevar a cabo este artículo.

\section{Conflicto de intereses}

Los autores declaran no tener ningún conflicto de intereses.

\section{Responsabilidades éticas}

Protección de personas y animales. Los autores declaran que para esta investigación no se han realizado experimentos en seres humanos ni en animales.

Confidencialidad de los datos. Los autores declaran que han seguido los protocolos de su centro de trabajo sobre la publicación de datos de pacientes.

Derecho a la privacidad y consentimiento informado. Los autores han obtenido el consentimiento informado de los pacientes y/o sujetos referidos en el artículo. Este documento obra en poder del autor de correspondencia.

\section{Bibliografía}

1. Reynolds W. The first laparoscopic cholecystectomy. JSLS. 2001;5:89-94.

2. Jacobs M, Verdeja JC, Goldstein HS. Minimally invasive colon resection (laparoscopic colectomy). Surg Laparosc Endosc. 1991;1:144-50.

3. Bonjer HJ, Hop WC, Nelson H, Sargent DJ, Lacy AM, Castells A, et al.; Transatlantic Laparoscopically Assisted vs Open Colectomy Trials Study Group. Laparoscopically assisted vs open colectomy for colon cancer: a meta-analysis. Arch Surg. 2007;142:298-303.

4. Hand-assisted laparoscopic surgery vs standard laparoscopic surgery for colorectal disease: a prospective randomized trial. HALS Study Group. Surg Endosc. 2000;14:896-901.

5. Guillou PJ, Quirke P, Thorpe H, Walker J, Jayne DG, Smith AM, et al.; MRC CLASICC trial group. Short-term endpoints of conventional versus laparoscopic-assisted surgery in patients with colorectal cancer (MRC CLASICC trial): multicentre, randomised controlled trial. Lancet. 2005;365:1718-26.

6. Leung KL, Kwok SP, Lam SC, Lee JF, Yiu RY, Ng SS, et al. Laparoscopic resection of rectosigmoid carcinoma: prospective randomised trial. Lancet. 2004;363:1187-92. 


\section{Andrada, et al.: Seguridad y reproducibilidad de la cirugía colorrectal}

7. Veldkamp R, Kuhry E, Hop WC, Jeekel J, Kazemier G, Bonjer HJ, et al.; COlon cancer Laparoscopic or Open Resection Study Group (COLOR) Laparoscopic surgery versus open surgery for colon cancer: short-term outcomes of a randomised trial. Lancet Oncol. 2005;6:477-84.

8. Braga M, Vignali A, Gianotti L, Zuliani W, Radaelli G, Gruarin P, et al. Laparoscopic versus open colorectal surgery: a randomized trial on short-term outcome. Ann Surg. 2002;236:759-66.

9. van der Pas $M H$, Haglind E, Cuesta MA, Fürst A, Lacy AM, Hop WC et al. Laparoscopic versus open surgery for rectal cancer (COLOR II): short-term outcomes of a randomised, phase 3 trial. Lancet Oncol. 2013;14:210-8

10. Schwenk W, Haase O, Neudecker J, Müller JM. Short term benefits for laparoscopic colorectal resection. Cochrane Database Syst Rev 2005;(3):CD003145.

11. Bennet C, Stryker S, Ferreria M, Adams J, Beart RW Jr. The learning curve for laparoscopic colorectal surgery. Preliminary results from a prospective analysis of 1194 laparoscopic-assisted colectomies. Arch Surg. 1997;132:41-4.

12. Schlachta C, Mamazza J, Seshadri P, Cadeddu M, Gregoire R, Poulin EC. Defining a learning curve for laparoscopic colorectal resections. Dis Colon Rectum. 2001;43:217-22.

13. Berends FJ, Kazemier G, Bonjer HJ, Lange JF. Subcutaneous metastases after laparoscopic colectomy. Lancet. 1994;344:58.

14. Martínez J, Targarona EM, Balagué C, Pera M, Trias M. Port site metastasis. An unresolved problem in laparoscopic surgery. A review. Int Surg. 1995;80:315-21.

15. Lacy AM, Delgado S, García-Valdecasas JC, Castells A, Piqué JM Grande L, et al. Port site metastases and recurrence after laparoscopic colectomy. A randomized trial. Surg Endosc. 1998:12:1039-42.

16. Dulskas A, Samalavicius NE, Gupta RK, Zabulis V. Laparoscopic colorectal surgery for colorectal polyps: single institution experience. Videosurgery Miniinv. 2015;10:73-8.

17. Gaertner WB, Kwaan MR, Madoff RD, Willis D, Belzer GE, Rothenberger DA, et al. The evolving role of laparoscopy in colonic diverticular disease: a systematic review. World J Surg. 2013;37:629-38.

18. Clinical Outcomes of Surgical Therapy Study Group. A comparison of laparoscopically assisted and open colectomy for colon cancer. $\mathrm{N}$ Engl J Med. 2004:350:2050-9.

19. Colon Cancer Laparoscopic or Open Resection Study Group, Buunen M, Veldkamp R, Hop WC, Kuhry E, Jeekel J, Haglind E, et al. Survival after laparoscopic surgery versus open surgery for colon cancer: long-term outcome of a randomised clinical trial. Lancet Oncol. 2009:10:44-52.

20. Hazebroek EJ; Color Study Group. COLOR: a randomized clinical tria comparing laparoscopic and open resection for colon cancer. Surg Endosc. 2002;16:949-53

21. Jacob BP, Salky B. Laparoscopic colectomy for colon adenocarcinoma: an 11-year retrospective review with 5-year survival rates. Surg Endosc. 2005; 19:643-9.

22. Nakamura $T$, Mitomi $H$, Ohtani $Y$, Kokuba $Y$, Sato $T$, Ozawa $H$, et al Comparison of long-term outcome of laparoscopic and conventional surgery for advanced colon and rectosigmoid cancer. Hepatogastroenterology. 2006:53:351-3.

23. Lacy AM, García-Valdecasas JC, Delgado S, Castells A, Taurá P Piqué JM, et al. Laparoscopy-assisted colectomy versus open colectomy for treatment of non-metastatic colon cancer: a randomised trial. Lancet. 2002;359:2224-9.
24. Jayne DG, Guillou PJ, Thorpe H, Quirke P, Copeland J, Smith AM, et al. Randomized trial of laparoscopic-assisted resection of colorectal carcinoma: 3-year results of the UK MRC CLASICC Trial Group. J Clin Oncol. 2007;25:3061-8.

25. Jayne DG, Thorpe HC, Copeland J, Quirke P, Brown JM, Guillou PJ. Five-year follow-up of the Medical Research Council CLASICC trial of laparoscopically assisted versus open surgery for colorectal cancer. $\mathrm{Br}$ J Surg. 2010:97:1638-45.

26. Kuhry E, Schwenk WF, Gaupset R, Romild U, Bonjer HJ. Long-term results of laparoscopic colorectal cancer resection. Cochrane Database Syst Rev 2008;(2):CD003432.

27. Bonjer HJ, Deijen $\mathrm{CL}$, Abis GA, Cuesta MA, van der Pas MH, de Lange-de Klerk ES, et al., COLOR II Study Group. A randomized trial of laparoscopic versus open surgery for rectal cancer. $\mathrm{N}$ Engl J Med. 2015;372:1324-32.

28. Arezzo A, Passera R, Salvai A, Arolfo S, Allaix ME, Schwarzer G, et al. Laparoscopy for rectal cancer is oncologically adequate: a systematic review and meta-analysis of the literature. Surg Endosc. 2015;29: 334-48.

29. Doyle DJ, Goyal A, Bansal P, Garmon EH. American Society of Anesthesiologists Classification. 2020 Jul 4. In: StatPearls Publishing ;2020 Jan-. PMID: 28722969. Disponible en: https://pubmed.ncbi.nlm.nih. gov/28722969/.

30. Dindo D, Demartines N, Clavien PA. Classification of surgical complications: a new proposal with evaluation in a cohort of 6,336 patients and results of a survey. Ann Surg. 2004;240:205-13.

31. Pascual M, Salvans S, Pera M. Laparoscopic colorectal surgery: current status and implementation of the latest technological innovations. World J Gastroenterol. 2016:22:704-17.

32. Surgical Care and Outcomes Assessment Program (SCOAP) Collaborative. Kwon S, Billingham R, Farrokhi E, Florence M, Herzig D, Horvath K, et al. Adoption of laparoscopy for elective colorectal resection: a report from the Surgical Care and Outcomes Assessment Program. J Am Coll Surg. 2012;214:909-18.e1.

33. Yeo H, Niland J, Milne D, ter Veer A, Bekaii-Saab T, Farma JM, et al. Incidence of minimally invasive colorectal cancer surgery at National Comprehensive Cancer Network centers. J Natl Cancer Inst. 2015;107:362.

34. Denost $Q$, Rouanet $P$, Faucheron JL. To drain or not to drain infraperitoneal anastomosis after rectal excision for cancer: the GRECCAR 5 Randomized Trial. Ann Surg. 2017;265:474-80.

35. den Dulk M, Noter SL, Hendriks ER, Brouwers MA, van der Vlies $\mathrm{CH}$, Oostenbroek RJ, et al. Improved diagnosis and treatment of anastomotic leakage after colorectal surgery. Eur J Surg Oncol. 2009;35:420-6.

36. Rahbari NN, Weitz J, Hohenberger W, Heald RJ, Moran B, Ulrich A, et al. Definition and grading of anastomotic leakage following anterior resection of the rectum: a proposal by the International Study Group of Rectal Cancer. Surgery. 2010;147:339-51.

37. Thorpe H, Jayne DG, Guillou PJ, Quirke P, Copeland J, Brown JM Medical Research Council Conventional versus Laparoscopic-Assisted Surgery In Colorectal Cancer Trial Group. Patient factors influencing conversion from laparoscopically assisted to open surgery for colorectal cancer. Br J Surg. 2008:95:199-205.

38. Fleshman J, Sargent DJ, Green E, Anvari M, Stryker SJ, Beart RW Jr, et al. Laparoscopic colectomy for cancer is not inferior to open surgery based on 5-year data from the COST Study Group trial. Ann Surg. 2007;246:655-62. 